\title{
PERTURBATION OF NORMAL OPERATORS ${ }^{1}$
}

\author{
S. L. JAMISON
}

The perturbation theory of linear operators concerns itself with the eigenvalues and eigenelements of a variable operator. If $T(\epsilon)$ is an operator depending on a real parameter $\epsilon$, the relation between the eigenvalues and eigenelements of the perturbed operator $T(\epsilon)(\epsilon \neq 0)$ and those of the unperturbed operator $T(0)$ is of particular interest. This theory finds considerable application in the field of quantum mechanics. In such applications the eigenvalues of an operator represent certain quantities which, by their very nature, must be real. It is therefore not surprising to find that emphasis has been given to self-adjoint operators. The first person to handle systematically the regular perturbation of self-adjoint operators was Franz Rellich [7]. ${ }^{2}$ By a different attack on the problem, Bela v. Sz. Nagy was able to improve on Rellich's theorem [5]. It was the conjecture of František Wolf that certain of these results would hold for normal operators-a class of operators which includes the self-adjoint. This paper substantiates that conjecture. Since the writing of this paper, Professor Wolf has rendered its main result a consequence of a general theory of analyticity for operators in a Banach space [9, section 6.4]. The proof given here exploits the relationship between a normal operator and a pair of self-adjoint operators.

A bounded linear transformation of an abstract Hilbert space $\mathfrak{S}$ into itself will be called an operator on $\mathfrak{S}$. The number $\lambda(\epsilon)$, the element $f(\epsilon)$ of $\mathfrak{S}$, and the operator $T(\epsilon)$ on $\mathfrak{S}$ will be called regular on the interval $|\epsilon|<\rho$ if they can be expressed as convergent power series in a real parameter $\epsilon$ :

$$
\begin{aligned}
\lambda(\epsilon) & =\lambda_{0}+\epsilon \lambda_{1}+\epsilon^{2} \lambda_{2}+\cdots, \\
f(\epsilon) & =f_{0}+\epsilon f_{1}+\epsilon^{2} f_{2}+\cdots, \\
T(\epsilon) & =T_{0}+\epsilon T_{1}+\epsilon^{2} T_{2}+\cdots,
\end{aligned}
$$

$\lambda_{k}, f_{k}$, and $T_{k}$ being respectively numbers, elements of $\mathfrak{E}$, and operators on $\mathfrak{S}$. Some mention should be made concerning the type of

Presented to the Society, December 27, 1950; received by the editors February 26, 1953 and, in revised form, June 25, 1953.

1 This paper is part of a thesis submitted by the author to the Graduate Division of the University of California (Summer, 1950) in partial satisfaction of the requirements for the $\mathrm{Ph} . \mathrm{D}$. degree. The author wishes to express his appreciation to Professor František Wolf, who directed the preparation of this paper.

${ }^{2}$ Numbers in brackets refer to the bibliography at the end of the paper. 
convergence involved in the series representation of regular elements and operators. Nagy, for example, requires strong (i.e. pointwise) convergence of the series $T(\boldsymbol{\epsilon})$. However, weak convergence is sufficient and, in this connection, actually equivalent to strong and uniform convergence. This is an immediate consequence of a theorem of Nelson Dunford [3, p. 6]: The weak analyticity of an operator $T(z)$ in a region implies its strong and uniform analyticity in this region. Similarly, the weak and strong convergence of $f(\epsilon)$ are equivalent concepts:

1. Reduction of a normal operator. A normal operator $N$ is one which commutes with its adjoint, i.e. $N N^{*}=N^{*} N$. In extending Rellich's theorem, the investigation of a normal operator will be reduced to the consideration of two self-adjoint operators. The following lemmas prepare the way for this reduction.

Lemma 1. If $\lambda$ is an eigenvalue of a normal operator $N$ and $\mathfrak{M}$ is the corresponding eigenspace, then $\bar{\lambda}$ is an eigenvalue of $N^{*}$ and $\mathfrak{M}$ is the corresponding eigenspace.

Proof. For $\phi \in \mathfrak{M}, N\left(N^{*} \phi\right)=N^{*}(N \phi)=N^{*}(\lambda \phi)=\lambda\left(N^{*} \phi\right)$. Thus $N^{*} \phi \in \mathfrak{M}$ and hence $\left(N^{*}-\bar{\lambda} I\right) \phi \in \mathfrak{M}$. But, for $f \in \mathfrak{M},\left(\left(N^{*}-\bar{\lambda} I\right) \phi, f\right)$ $=(\phi,(N-\lambda I) f)=0$ and hence $\left(N^{*}-\bar{\lambda} I\right) \phi$ is orthogonal to every element of $\mathfrak{M}$ and, in particular, to itself. Therefore $\left(N^{*}-\bar{\lambda} I\right) \phi=0$; that is, $N^{*} \phi=\bar{\lambda} \phi$ for each $\phi \in \mathfrak{M}$. Thus the eigenspace $\mathfrak{M}^{*}$ of $N^{*}$ corresponding to $\bar{\lambda}$ includes $\mathfrak{M}$. Since $N^{* *}=N$, this same proof yields the inclusion $\mathfrak{M}^{*} \subset \mathfrak{M}$. Thus $\mathfrak{M}^{*}=\mathfrak{M}$.

In what fo!lows, the spectrum of an operator $T$ will be denoted by $\sigma(T)$ and the point spectrum by $P \sigma(T)$.

Lemma 2. Let $N$ be a normal operator and let $N=A+i B$ where $A=\left(N+N^{*}\right) / 2$ and $B=\left(N-N^{*}\right) / 2 i$.

(a) If $\xi \in \sigma(N)$, then $\Re(\xi) \in \sigma(A)$ and $\Im(\xi) \in \sigma(B)$.

(b) If $\xi \in P \sigma(N)$, then $\Re(\xi) \in P \sigma(A)$ and $\Im(\xi) \in P \sigma(B)$.

(c) If $\alpha \in \sigma(A)$, then there is a $\beta$ in $\sigma(B)$ such that $(\alpha+i \beta) \in \sigma(N)$.

(d) If $\beta \in \sigma(B)$, then there is an $\alpha$ in $\sigma(A)$ such that $(\alpha+i \beta) \in \sigma(N)$.

(e) The eigenspace of $N$ corresponding to an eigenvalue $\xi$ is the intersection of the eigenspaces of $A$ and $B$ corresponding respectively to $\Re(\xi)$ and $\Im(\xi)$.

Proof. The operators $A$ and $B$ are clearly self-adjoint. Let $K, E_{1}$, and $E_{2}$ be the spectral measures (i.e. resolutions of the identity) corresponding to $N, A$, and $B$ respectively. Let $M_{1}$ represent the interval $x_{1}<x \leqq x_{2}, M_{2}$ the interval $y_{1}<y \leqq y_{2}$, and $M$ the rectangle $x_{1}<\Re(\xi)$ 
$\leqq x_{2}, y_{1}<\Im(\xi) \leqq y_{2}$. Then

$$
K(M)=E_{1}\left(M_{1}\right) E_{2}\left(M_{2}\right) .
$$

That is, the spectral measure of $N$ on the rectangle $M$ of the complex plane is the product of the spectral measures of $A$ and $B$ on the real intervals $M_{1}$ and $M_{2}$ respectively [2, p. 72].

Suppose now that $\xi \in \sigma(N)$ and choose $M$ so as to include the point $\xi=x+i y$ as an interior point. Then $K(M) \neq 0[6$, p. 54] and therefore, from (1), $E_{1}\left(M_{1}\right) \neq 0$ and $E_{2}\left(M_{2}\right) \neq 0$. Hence $M_{1}$ contains a point of $\sigma(A)$ and $M_{2}$ contains a point of $\sigma(B)\left[8\right.$, p. 184]. Since $x_{1}$ and $y_{1}$ can be taken arbitrarily close to $x_{2}$ and $y_{2}$ respectively, it follows that $\Re(\xi) \in \sigma(A)$ and $\Im(\xi) \in \sigma(B)$.

Suppose that $\xi=\left(x_{2}+i y_{2}\right) \in P \sigma(N)$. Then $K$ has a discontinuity at $\xi$. That is, $K(M)$ does not tend to zero as $x_{1} \rightarrow x_{2}$ and $y_{1} \rightarrow y_{2}$. Because of (1), $E_{1}\left(M_{1}\right)$ does not tend to zero as $x_{1} \rightarrow x_{2}$ and $E_{2}\left(M_{2}\right)$ does not tend to zero as $y_{1} \rightarrow y_{2}$. That is, $x_{2}$ and $y_{2}$ are points of discontinuity of $E_{1}$ and $E_{2}$ respectively. Thus $x_{2}=\Re(\xi) \in P \sigma(A)$ and $y_{2}=\Im(\xi)$ $\in P \sigma(B)$.

The boundedness of $N$ implies the boundedness of both $A$ and $B$. It is therefore possible to take $M_{2}$ so as to include all of $\sigma(B)$. With such a choice for $M_{2}, E_{2}\left(M_{2}\right)=I$ and (1) becomes

$$
K(M)=E_{1}\left(M_{1}\right) .
$$

If $\alpha \in \sigma(A)$ and if $M_{1}$ includes $\alpha$ as an interior point, then $E_{1}\left(M_{1}\right) \neq 0$ and hence $K(M) \neq 0$. $M$ therefore contains a point of $\sigma(N)$. Since the interval $M_{1}$ can be made to extend arbitrarily little on each side of $\alpha$, it follows that there is some $\xi$ with $\Re(\xi)=\alpha$ in $\sigma(N)$. Then, by part (a), $\Im(\xi) \in \sigma(B)$. This proves (c); (d) is proved similarly. If the Hilbert space involved is finite-dimensional, the spectrum of any operator consists entirely of point spectrum. In this case, therefore, if $\alpha \in P \sigma(A)$ there is a $\beta \in P \sigma(B)$ such that $(\alpha+i \beta) \in P \sigma(N)$.

Let $\xi$ be an eigenvalue of $N$ and let $Q=\{\phi \mid N \phi=\xi \phi\}, R$ $=\{\phi \mid A \phi=\Re(\xi) \phi\}$, and $S=\{\phi \mid B \phi=\Im(\xi) \phi\}$. By Lemma $1, Q$ is also equal to $\left\{\phi \mid N^{*} \phi=\xi \phi\right\}$. Thus, if $\phi \in Q, \quad(A-\Re(\xi) I) \phi$ $=\left(\left(N+N^{*}\right) / 2-(\xi+\bar{\xi}) I / 2\right) \phi=(N-\xi I) \phi / 2+\left(N^{*}-\bar{\xi} I\right) \phi / 2=0$ and, similarly, $(B-\Im(\xi) I) \phi=0$. Therefore, $\phi \in Q$ implies $\phi \in R$ and $\phi \in S$; that is, $Q \subseteq R \cap S$. If $\phi \in R \cap S$, then $(N-\xi I) \phi=((A+i B)-(\Re(\xi)$ $+i \Im(\xi)) I) \phi=(A-\Re(\xi) I) \phi+i(B-\Im(\xi) I) \phi=0$. Thus $\phi \in R \cap S$ implies $\phi \in Q$ and hence $R \cap S \subseteq Q$. Therefore $Q=R \cap S$ and the lemma is proved.

2. The operators $P$ and $U$. If $T$ is an operator, a set $R$ of complex numbers will be called $T$-admissible if it consists of a finite number of 
open connected sets which are mutually disjoint and if the boundary $\Gamma$ of $R$ consists of a finite number of disjoint simple closed curves of finite length all lying in the resolvent set of $T$. Such a curve $\Gamma$ will likewise be called $T$-admissible. If $\Gamma$ is $T$-admissible, Dunford [1, pp. 196-197] has proved that

$$
P=\frac{-1}{2 \pi i} \int_{\Gamma}(T-z I)^{-1} d z
$$

is a bounded idempotent reducing $T$ and that $T$, considered as an operator on $P \mathfrak{S}$, has for its spectrum the spectral set of $T$ which is enclosed by $\Gamma$ (i.e., $\sigma(T) \cap R$ ). This result prepares the way for the following lemma.

Lemma 3. Let $N(\epsilon)=N_{0}+\epsilon N_{1}+\epsilon^{2} N_{2}+\cdots$ be regular and normal for $|\epsilon|<\rho$ and let $\Gamma$ be $N_{0}$-admissible. Then there is a $\rho^{\prime}>0$ such that, for $|\epsilon|<\rho^{\prime}$, the operator

$$
P(\epsilon)=\frac{-1}{2 \pi i} \int_{\Gamma}(N(\epsilon)-z I)^{-1} d z
$$

is a regular projection on the subspace $\mathfrak{M}(\boldsymbol{\epsilon})$ of $\mathfrak{W}$ which corresponds to that part of the spectrum of $N(\epsilon)$ which is enclosed by $\Gamma$. That is,

$$
P(\epsilon)=\int_{R} d K_{\xi}(\epsilon)
$$

where $K_{\xi}(\epsilon)$ is the spectral measure corresponding to $N(\epsilon)$. The dimension of $\mathfrak{M}(\epsilon)$ is independent of $\epsilon . P(\epsilon)$ reduces $N(\epsilon)$ and $N(\epsilon)$, considered as an operator on $\mathfrak{M}(\epsilon)$, has $R \cap \sigma(N(\epsilon))$ as its spectrum.

This lemma was proved by Nagy [5, pp. 351-356] for the case where $N(\epsilon)$ is self-adjoint. Only minor modifications in that proof are necessary to render it applicable to normal operators. The next result is likewise due to Nagy [5, p. 350].

Lemma 4. Let $P$ and $Q$ be projections on manifolds $\mathfrak{M}$ and $\mathfrak{N}$ of $\mathfrak{E}$ and let $\|Q-P\|<1$. Then the operator

$$
U=Q[I+P(Q-P) P]^{-1 / 2} P
$$

is partially isometric with initial set $\mathfrak{M}$ and final set $\mathfrak{R}$.

The following properties of $U$ are a consequence of this lemma:
(a) $U$ maps $\mathfrak{M}$ onto $\mathfrak{N}$ and $U^{*}$ maps $\mathfrak{R}$ onto $\mathfrak{M}$,
(b) $U^{*} U=P$ and $U U^{*}=Q$. 
3. Rellich's Theorem for a normal operator. We are now in a position to extend one of Rellich's main results. We first state the theorem as proved by Rellich for self-adjoint operators [7, p. 610] and then extend it so as to include normal operators.

Rellich's Theorem. Let $A(\epsilon)=A_{0}+\epsilon A_{1}+\epsilon^{2} A_{2}+\cdots$ be regular and self-adjoint for $|\epsilon|<\rho$ and let $\lambda_{0}$ be an eigenvalue of $A_{0}$ of finite multiplicity $m$; suppose, moreover, that the interval $\lambda_{0}-d_{1}<\lambda<\lambda_{0}$ $+d_{2}\left(d_{1}>0, d_{2}>0\right)$ does not contain any other points of $\sigma\left(A_{0}\right)$. Then, for $\epsilon$ sufficiently small, there are $m$ regular numbers

$$
\lambda^{i}(\epsilon)=\lambda_{0}+\epsilon \lambda_{1}^{i}+\epsilon^{2} \lambda_{2}^{i}+\cdots
$$

and $m$ regular, normed, mutually orthogonal elements

$$
\phi^{i}(\epsilon)=\phi_{0}^{i}+\epsilon \phi_{1}^{i}+\epsilon^{2} \phi_{2}^{i}+\cdots
$$

such that $A(\epsilon) \phi^{i}(\epsilon)=\lambda^{i}(\epsilon) \phi^{i}(\epsilon)$ for $i=1,2, \cdots, m$. If $d_{1}^{\prime}$ and $d_{2}^{\prime}$ are positive with $d_{1}^{\prime}<d_{1}$ and $d_{2}^{\prime}<d_{2}$, then there is a number $\rho^{\prime}$ such that, for $|\epsilon|<\rho^{\prime}$, the spectrum of $A(\epsilon)$ in the interval $\lambda_{0}-d_{1}^{\prime}<\lambda<\lambda_{0}+d_{2}^{\prime}$ consists exactly of the points $\lambda^{1}(\epsilon), \cdots, \lambda^{m}(\epsilon)$.

Now let $N(\epsilon)=N_{0}+\epsilon N_{1}+\epsilon^{2} N_{2}+\cdots$ be a regular normal operator for $|\epsilon|<\rho$. Let $\lambda_{0}$ be an eigenvalue of $N_{0}$ of finite multiplicity $m$ and suppose that $\lambda_{0}$ is the only point of $\sigma\left(N_{0}\right)$ in the circle $\left|z-\lambda_{0}\right|$ $<2 d$. Let $\Gamma$ be the circle $\left|z-\lambda_{0}\right|=d$ and let $P(\epsilon)=P_{0}+\epsilon P_{1}+\epsilon^{2} P_{2}$ $+\cdots$ be the projection of Lemma 3. Since $P(\epsilon)$ is regular we can choose $\rho^{\prime}$ so small that, for $|\epsilon|<\rho^{\prime},\left\|P(\epsilon)-P_{0}\right\|<1$ and

$$
\begin{aligned}
{\left[I+P_{0}\left(P(\epsilon)-P_{0}\right) P_{0}\right]^{-1 / 2} } & =\sum_{\nu=0}^{\infty}\left(\begin{array}{c}
-1 / 2 \\
\nu
\end{array}\right)\left[P_{0}\left(P(\epsilon)-P_{0}\right) P_{0}\right]^{\nu} \\
& =\sum_{\nu=0}^{\infty}\left(\begin{array}{c}
-1 / 2 \\
\nu
\end{array}\right)\left[\sum_{k=1}^{\infty} \epsilon^{k} P_{0} P_{k} P_{0}\right]^{\nu}
\end{aligned}
$$

is regular. Hence, by Lemma 4, the operator $U(\epsilon)=P(\epsilon)\left[I+P_{0}(P(\epsilon)\right.$ $\left.\left.-P_{0}\right) P_{0}\right]^{-1 / 2} P_{0}$ is regular and partially isometric with initial set $\mathfrak{M}_{0}$ and final set $\mathfrak{M}(\epsilon)$.

Let $C(\epsilon)=U^{*}(\epsilon) N(\epsilon) U(\epsilon)$. By (3)(b), $U(\epsilon) U^{*}(\epsilon)=P(\epsilon)$ and, by Lemma 3, $P(\epsilon)$ commutes with $N(\epsilon)$ and hence with $N^{*}(\epsilon)$. Thus $C^{*}(\boldsymbol{\epsilon}) C(\boldsymbol{\epsilon})=U^{*}(\boldsymbol{\epsilon}) N^{*}(\boldsymbol{\epsilon}) U(\boldsymbol{\epsilon}) U^{*}(\boldsymbol{\epsilon}) N(\boldsymbol{\epsilon}) U(\boldsymbol{\epsilon})=U^{*}(\boldsymbol{\epsilon}) N^{*}(\boldsymbol{\epsilon}) P(\boldsymbol{\epsilon}) N(\boldsymbol{\epsilon}) U(\boldsymbol{\epsilon})$ $=U^{*}(\boldsymbol{\epsilon}) N^{*}(\boldsymbol{\epsilon}) N(\boldsymbol{\epsilon}) P(\boldsymbol{\epsilon}) U(\boldsymbol{\epsilon})=U^{*}(\boldsymbol{\epsilon}) N(\boldsymbol{\epsilon}) N^{*}(\boldsymbol{\epsilon}) P(\boldsymbol{\epsilon}) U(\boldsymbol{\epsilon})=U^{*}(\boldsymbol{\epsilon}) N(\boldsymbol{\epsilon})$ $P(\epsilon) N^{*}(\epsilon) U(\epsilon)=U^{*}(\epsilon) N(\epsilon) U(\epsilon) U^{*}(\epsilon) N^{*}(\epsilon) U(\epsilon)=C(\epsilon) C^{*}(\epsilon)$ and hence $C(\epsilon)$ is normal. By (3) (a) and Lemma 3, $U(\epsilon)$ maps $\mathfrak{M}_{0}$ onto $\mathfrak{M}(\boldsymbol{\epsilon}), N(\epsilon)$ maps $\mathfrak{M}(\boldsymbol{\epsilon})$ into $\mathfrak{M}(\boldsymbol{\epsilon})$, and $U^{*}(\boldsymbol{\epsilon})$ maps $\mathfrak{M}(\boldsymbol{\epsilon})$ onto $\mathfrak{M}_{0}$. 
Thus $C(\boldsymbol{\epsilon})$ may be considered as a regular normal operator on $\mathfrak{M}_{0}$. Let $D(\epsilon)$ be the restriction of $C(\boldsymbol{\epsilon})$ to $\mathfrak{M}_{0}$. Since the restriction of a normal operator to a manifold which reduces it is normal [6, p. 34], $D(\epsilon)$ is a regular normal operator on $\mathfrak{M}_{0}$.

Let $f \in \mathbb{M}_{0}$. Then $f=P_{0} f=U^{*}(\boldsymbol{\epsilon}) U(\boldsymbol{\epsilon}) f$ by $(3)(\mathrm{b})$ and so $(D(\boldsymbol{\epsilon})$ $-z I) f=(C(\boldsymbol{\epsilon})-z I) f=\left(U^{*}(\boldsymbol{\epsilon}) N(\boldsymbol{\epsilon}) U(\boldsymbol{\epsilon})-z I\right) f=U^{*}(\boldsymbol{\epsilon})(N(\boldsymbol{\epsilon})-z I) U(\boldsymbol{\epsilon}) f$; that is,

$$
(D(\epsilon)-z I) f=U^{*}(\epsilon)(N(\epsilon)-z I) U(\epsilon) f \quad \text { for } f \in \mathfrak{M}_{0} .
$$

Let $z$ be an eigenvalue of $D(\epsilon)$ and let $f \neq 0$ be a corresponding eigenelement in $\mathfrak{M}_{0}$. Then, by $(4), U^{*}(\epsilon)(N(\epsilon)-z I) U(\epsilon) f=0$ and hence $0=U(\boldsymbol{\epsilon}) U^{*}(\boldsymbol{\epsilon})(N(\boldsymbol{\epsilon})-z I) U(\boldsymbol{\epsilon}) f=P(\boldsymbol{\epsilon})(N(\boldsymbol{\epsilon})-z I) U(\boldsymbol{\epsilon}) f=(N(\boldsymbol{\epsilon})-z I)$ $U(\epsilon) f$. Thus $z$ is an eigenvalue of $N(\boldsymbol{\epsilon})$ and $U(\epsilon) f$ is a corresponding eigenelement; that is,

$$
D(\epsilon) f=z f \quad \text { implies } N(\epsilon) U(\epsilon) f=z U(\epsilon) f .
$$

Suppose now that $N(\boldsymbol{\epsilon})$, regarded as an operator on $\mathfrak{M}(\boldsymbol{\epsilon})$, has $z$ as an eigenvalue and that $g \neq 0$ is a corresponding eigenelement in $\mathfrak{M}(\boldsymbol{\epsilon})$. Let $f=U^{*}(\boldsymbol{\epsilon}) g$. Then $f \in \mathfrak{M}_{0}$ and, since $U(\boldsymbol{\epsilon}) f=U(\boldsymbol{\epsilon}) U^{*}(\boldsymbol{\epsilon}) g=P(\boldsymbol{\epsilon}) g$ $=g \neq 0, f \neq 0$. Thus, by (4),

$$
(D(\epsilon)-z I) f=U^{*}(\boldsymbol{\epsilon})(N(\boldsymbol{\epsilon})-z I) g=0
$$

and hence, for $g \in \mathfrak{M}(\epsilon)$,

$$
N(\epsilon) g=z g \text { implies } D(\epsilon) U^{*}(\epsilon) g=z U^{*}(\epsilon) g .
$$

Since $\mathfrak{M}_{0}$ and $\mathfrak{M}(\boldsymbol{\epsilon})$ have finite dimension $m$, the entire spectra of $D(\epsilon)$ and of the restriction of $N(\boldsymbol{\epsilon})$ to $\mathfrak{M}(\boldsymbol{\epsilon})$ consist of eigenvalues. In the light of Lemma 3, therefore, results (5) and (6) yield

$$
\sigma(D(\epsilon))=\sigma(N(\epsilon)) \cap R
$$

where $R$ is the interior of $\Gamma$. In particular $\sigma(D(0))=\lambda_{0}$.

Let $A(\boldsymbol{\epsilon})=\left(D(\boldsymbol{\epsilon})+D^{*}(\boldsymbol{\epsilon})\right) / 2$ and $B(\boldsymbol{\epsilon})=\left(D(\boldsymbol{\epsilon})-D^{*}(\boldsymbol{\epsilon})\right) / 2 i . \quad A(\boldsymbol{\epsilon})$ and $B(\epsilon)$ are regular self-adjoint operators. By Lemma 2 , the spectra of $A_{0}$ and $B_{0}$ consist of the single points $\Re\left(\lambda_{0}\right)$ and $\Im\left(\lambda_{0}\right)$ respectively, each with multiplicity $m$. We can then apply Rellich's Theorem and claim the existence of $2 m$ regular numbers

$$
\begin{aligned}
\alpha^{k}(\epsilon) & =\Re\left(\lambda_{0}\right)+\epsilon \alpha_{1}^{k}+\epsilon^{2} \alpha_{2}^{k}+\cdots, & k & =1,2, \cdots, m, \\
\beta^{j}(\epsilon) & =\Im\left(\lambda_{0}\right)+\epsilon \beta_{1}^{j}+\epsilon^{2} \beta_{2}^{j}+\cdots, & j & =1,2, \cdots, m,
\end{aligned}
$$

which constitute the entire spectra of $A(\epsilon)$ and $B(\epsilon)$ respectively. By Lemma 2, the $\alpha^{k}(\boldsymbol{\epsilon})$ and $\beta^{j}(\boldsymbol{\epsilon})$ can be paired off so that $\alpha^{k}(\boldsymbol{\epsilon})$ 
$+i \beta^{k}(\epsilon) \in \sigma(D(\epsilon))$. Since $\mathfrak{M}_{0}$ is $m$-dimensional, the $m$ (not necessarily distinct) numbers

$$
\lambda^{k}(\epsilon)=\alpha^{k}(\epsilon)+i \beta^{k}(\epsilon)=\lambda_{0}+\epsilon\left(\alpha_{1}^{k}+i \beta_{1}^{k}\right)+\epsilon^{2}\left(\alpha_{2}^{k}+i \beta_{2}^{k}\right)+\cdots
$$

are eigenvalues of $D(\epsilon)$ and constitute the entire spectrum of $D(\epsilon)$. By (7), the $\lambda^{k}(\epsilon)$ are also eigenvalues of $N(\epsilon)$ and, in fact, constitute the only part of $\sigma(N(\epsilon))$ inside $\Gamma$. Now the spectrum of an operator moves continuously with the operator $\left[4\right.$, p. 118]. Since $\lambda_{0}$ was assumed to be the only part of $\sigma\left(N_{0}\right)$ in the circle $\left|z-\lambda_{0}\right|<2 d$, for any $\eta, 0<\eta<d$, there is a $\gamma>0$ such that the spectrum of $N(\epsilon)$ in the circle $\left|z-\lambda_{0}\right|<2 d-\eta$ consists exactly of the $m$ points $\lambda^{k}(\epsilon)$ provided that $|\epsilon|<\gamma$.

Finally we must prove the existence of regular eigenelements corresponding to the $\lambda^{k}(\epsilon)$. If the multiplicity of the eigenvalue $\lambda_{0}$ is $m=1$, then $\mathfrak{M}_{0}$ is one-dimensional and hence the eigenspace of $A(\epsilon)$, $B(\epsilon)$, and $D(\epsilon)$ are all one-dimensional-in fact, they all coincide with $\mathfrak{M}_{0}$. Let $f$ be a normed element of $\mathfrak{M}_{0}$. Since every nonzero element of the one-dimensional space $\mathfrak{M}_{0}$ is an eigenelement of every operator on $\mathfrak{M}_{0}, f$ is an eigenelement of $D(\epsilon)$ and hence, by (5), $U(\epsilon) f$ is a regular eigenelement of $N(\epsilon)$ corresponding to $\lambda(\epsilon)=\lambda_{0}+\epsilon \lambda_{1}+\epsilon^{2} \lambda_{2}+\cdots$ and $\|U(\epsilon) f\|=\|f\|=1$. The proof now goes by induction with respect to the multiplicity $m$. This induction for the normal case is essentially the same as given by Nagy [5, pp. 360-362] for the self-adjoint case and will therefore be omitted here.

The preceding arguments constitute the proof of Rellich's Theorem for normal operators:

Theorem. Let $N(\epsilon)=N_{0}+\epsilon N_{1}+\epsilon^{2} N_{2}+\cdots b e$, for $|\epsilon|<\rho$, a regular normal operator on a Hilbert space $\mathfrak{S}$. Let $\lambda_{0}$ be an eigenvalue of $N_{0}$ of finite multiplicity $m$ and suppose that the open circle $\left|z-\lambda_{0}\right|<2 d$ does not contain any other points of $\sigma\left(N_{0}\right)$. Then there exist $m$ numbers $\lambda^{k}(\boldsymbol{\epsilon})$ and $m$ elements $\phi^{k}(\boldsymbol{\epsilon})$ of $\mathfrak{W}$,

$$
\begin{aligned}
& \lambda^{k}(\epsilon)=\lambda_{0}+\epsilon \lambda_{1}^{k}+\epsilon^{2} \lambda_{2}^{k}+\cdots, \\
& \phi^{k}(\epsilon)=\phi_{0}^{k}+\epsilon \phi_{1}^{k}+\epsilon^{2} \phi_{2}^{k}+\cdots,
\end{aligned}
$$

which are regular on some interval $|\epsilon|<\delta$ and such that the $\lambda^{k}(\epsilon)$ are eigenvalues of $N(\epsilon)$ and the $\phi^{k}(\epsilon)$ are corresponding eigenelements. The $\phi^{k}(\epsilon)$ form, for fixed $\epsilon$, an orthonormal set. To each $\eta, 0<\eta<d$, there corresponds a $\gamma>0$ such that, for $|\epsilon|<\gamma$, the spectrum of $N(\epsilon)$ which is in the circle $\left|z-\lambda_{0}\right|<2 d-\eta$ consists exactly of the $m$ points $\lambda^{k}(\epsilon)$-each eigenvalue being counted as often as its multiplicity. 


\section{BIBLIOGRAPHY}

1. N. Dunford, Spectral theory. I. Convergence to projections, Trans. Amer. Math. Soc. vol. 54 (1943) pp. 185-217.

2. P. Halmos, Introduction to Hilbert space, Chelsea, 1951.

3. E. Hille, Notes on linear transformations, Ann. of Math. (2) vol. 40 (1939) pp. $1-47$.

4. - Functional analysis and semi-groups, Amer. Math. Soc. Colloquium Publications, vol. 31, 1948.

5. B. v. Sz. Nagy, Perturbations des transformations autoadjointes dans l'espace de Hilbert, Comment. Math. Helv. vol. 19 (1946) pp. 347-366.

6. - Spektraldarstellung linearer Transformationen des Hilbertschen Raumes, Springer, 1942.

7. F. Rellich, Störungstheorie der Spektralzerlegung, Math. Ann. vol. 113 (1937) pp. 600-619.

8. M. Stone, Linear transformations in Hilbert space, Amer. Math. Soc. Colloquium Publications, vol. 15, 1932.

9. F. Wolf, Analytic perturbation of operators in Banach spaces, Math. Ann. vol. 124 (1952) pp. 317-333.

University of California, Berkeley 